\title{
Creating Interactive Environments for Education
}

\author{
Ramirez-Velarde, Raul V. ${ }^{1}$, Garcia-Rueda, Jose J. ${ }^{2}$ and Alexandrov, Nia ${ }^{3}$ \\ ${ }^{1}$ ITESM, Eugenio Garza Sada 2501, Monterrey, N.L., Mexico \\ rramirez@itesm.mx \\ ${ }^{2}$ Universidad Carlos III Madrid, Av. De la Universidad 30, Leganes, Spain, \\ rueda@uc3m.edu.es \\ ${ }^{3}$ The University of Reading, PO Box 68, Reading RG6 6BX, Berkshire, United Kingdom \\ n.s.alexandrov@reading.ac.uk
}

\begin{abstract}
In this paper, based on recent scientific findings in the fields of neurology, evolutionary psychology and cognitive psychology we propose a software architecture and technology supported learning activities model.
\end{abstract}

Keywords: Educational technology, human-computer interaction, interactivity, integrated learning environments.

\section{Introduction}

Now days our classrooms are beginning to fill with all kinds of technology applied to learning. Those resources support learning activities that help students acquire different skills, abilities, attitudes, and knowledge. Some resources focus on perception, some focus on reflection, some focus on reasoning, some focus on planning, some focus on communication and some others focus on action.

The E-LANE project has produced a table of suggested resources that can be applied to different skills [1]. Problem solving, the third skill level en E-LANE's Educational Model, is about action, thus a learning environment that aims to teach such a skill would need to be able to map computer actions to real world actions. We call such mapped human-computer dialog Interaction.

Interactive educational software can be enhanced through the clever use of images and interactions that enable students develop skills through several levels and several learning styles, specifically problem solving skills [2], [3]. We call such software environments Interactive Learning Environments (ILE). In order to be effective learning tools, an ILE must produce the necessary scaffolding to help students complete the learning cycle using different learning channels [4]. Thus in this paper we aim to describe the general characteristics of effective ILEs that can help students learn conceptual, contextual, procedural and problem solving skills. 


\section{New Science of the Brain}

\subsection{Natural Learning}

Neuroscience has made many advances in recent years that can be applied to improve teaching and learning. One of the most promising applications of neuroscience to learning is James Zulls "Natural Learning” [5]. Natural Leaning links functional parts of the brain to David Kolb’s [6] learning cycle (See Fig. 1). Fig. 1 explains the relationships between the cerebral cortex and the learning cycle.

As the Fig. 1 shows, Kolbs's Natural Learning Cycle (NLC) is in complete agreement with the structure and function of the brain. When we learn, we usually first perceive the information. This information is integrated to previous knowledge acquired by past experience. We later use the newly acquired knowledge to solve problems, create plans for action, to act upon such plans in such a way that a test of our skills is created. The result is perceived by our senses and the cycle begins again.

\subsection{The Potential of All}

The human brain is an organ for survival. Humans sense, integrate, abstract and act as a mechanism for survival [5], [7]. Human beings developed highly sophisticated brains that feel two urges: Understanding and control [8]. As indicated by NLC, the purpose of the brain is to establish a mental abstraction of the world that will enable our body to act. An ILE helps students to understand the world and control learning.

\section{The Need for Interactive Learning Environments}

\subsection{Characteristics of ILE}

The advantages that ILEs have over traditional media and other educational digital media are better visualization, better navigation, increased interaction, and reduced cognitive overload.

Better Visualization. An ILE can improve visualization as compared to other media by using animation, 2D/3D navigation and exploration and/or slideshows to show information form different angles and different times. It can use visualization techniques to create useful schemas [9], [10] to map the real world into the brain as well as use metaphors to map real-world actions into the virtual environment. The visualization techniques used can be as low tech as simple text or simple line artwork, 2D graphics [11], 3D graphics [12], [13], [14] and stereoscopic 3D [15].

Better Navigation. An ILE can remember all the pages that a student has reviewed; it can show two pages at the same time in different windows; it can provide 
hundreds of bookmarks; it can link different interrelated parts, it can provide arbitrary searches and it can make decisions based on student performance or preferences.

Better Interaction. We define interaction as a meaningful gesture which is equivalent, through a metaphor, to a physical action in the real world. Interaction is what an ILE would naturally do best. Sigital materials create virtual environments (2D or 3D) that mimic the real world and provide high transferability. Using our guidelines, many types of visualization-interaction sets can be used to create an ILE.

Lower Cognitive Load. Usually, students migrate from learning environments in order to do all the tasks they need to achieve learning.. In each of those environments they need to learn how to use them, how to behave, how to relate them and are subject to all kinds of distractions. Of all the things that a learner must learn in order to learn, very little might be intrinsic to the particular learning goal. An ILE should also be integrated, which means that the learner is supported through the entire learning cycle.

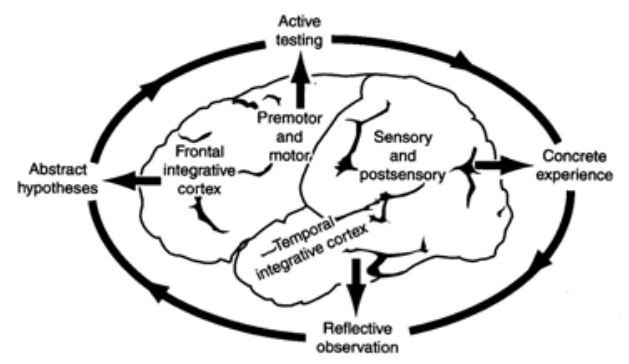

Fig. 1. Kolb’s Natural Leaning Cycle and the Human Brain. Taken from [5]

\subsection{Working Memory and Cognitive Load}

When we learn, human beings exercise two types of memory working memory and long-term memory. Working memory is short term and very limited. It holds perceived information and processes it before it is transferred to long-term memory [16]. It was designed by evolution to be very volatile so human beings could focus alternatively in different signals coming through our senses [5]. Long-term memory holds information by constructing neuronal networks [5] which are perceived as cognitive schemata by psychologists [10]. The cognitive load is the amount of work required of the working memory in order to learn [9]. Consequently, if instruction is delivered in such a way that working memory cannot adequately process it, it is said that it produces cognitive overload (CO) [16]. As people learn, perception is related to stored knowledge and already existent neuronal networks become stronger [5], [16], promoting automated responses that free capacity in working memory [16].

There are three types of cognitive load [10], [16]: Intrinsic cognitive load (ICL) is related to the inherent complexity of the subject and it is only influenced by the level of expertise of the learner. Extraneous cognitive load (ECL) is caused by factors that are not essential to the content, such as presentation method, interactions with the 
environment (real or digital) or activities that split attention between multiple sources of information. Extraneous cognitive load should be minimized as much as possible. Germane cognitive load (GCL) is related to the transition from novice to expert by helping learners to acquire schemata and automation. GCL has also being related to interest and motivation because both are needed to keep learners working on building stronger neuronal networks once they have past the initial phase of perception.

The NLC is consistent with CLT; ICL relates to frontal cortex and the ability to hypothesize and plan, ECL relates to noise perceived through the sensory and postsensory cortex that channels concrete experiences, whereas GCL relates to temporal integrative cortex or reflective observation. The NLC ensures transfer of learning to real life. It also establishes a path of learning activities that insure that skills acquired by students through ILE will transfer to real-life.

\subsection{Multimedia Simulation as ILEs}

The typical example of an ILE is as a multimedia-simulation. This would consist of short video/animation/audio/text presentations followed by a simulation of the real environment. According to the NLC, the general algorithm would be as follows:

1. Segment each learning unit to its minimal expression (reduce CL). That is, concentrate on what is important. Leave every thing else as optional [16], [9].

2. Establish an appropriate visualization technique based on human perception of shape, texture and location [17], [18], [19].

3. Produce short animations or video to show procedures [20].

4. Show several examples. Make some of them worked examples (an example in which the some of the solution is shown) [20], [22].

5. Ask students to complete solutions to partially solved problems or fill data organization forms of increasing difficulty (this is called scaffolding) [4].

6. Direct students to free-goal simulations that will allow students use problem solving strategies. Drill and practice varying types of problems [4], [5].

\section{Architecture for Interactive Learning Environments}

Drawing on CLT and NLC we propose that an ILE is actually a very simple artifact constructed by three modules: View, Control and Model (See Fig. 2).

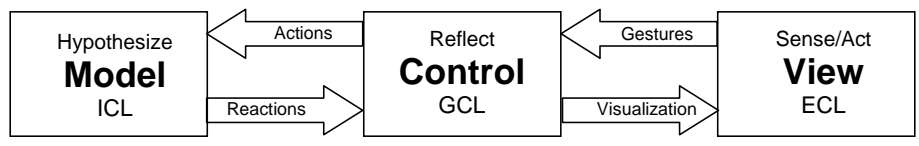

Fig. 2. ILEs Module Architecture 


\subsection{View Module}

This module presents the user interface. It captures user input and renders model visualization, that is, the actual delivery of lessons following certain visual formats. It engages the premotor, motor, sensory and postsensory parts of the brain. The View module uses visualization techniques for two purposes: to reduce ECL (user interface intuitive and appealing) and to improve transfer to real life. This can be achieved by using metaphors and similes that allow a learner to use already stored neuronal networks to understand what he is seeing and what she is doing, that is schemata.

\subsection{Model Module}

This an abstraction of the real world mapped into a computer model. This module tracks the state of the program storing the most important information computed during the learning process or captured form the student. Naturally, in a learning environment it would be responsible of tracking student progress, state of current chapter, provide context sensitive feedback and lesson flow possibilities. It must be able to present content in different levels of complexity and as such, its main responsibility is to manage ICL. Although the view module must visualize user interface concepts and actions, it is usually the model module's job to deal with concepts, relations and skills visualization.

\subsection{Control Module}

It maps gestures and information received from students and translates them to actions upon model state.

This module is responsible for managing GCL. Through a careful task analysis, the control module is programmed to understand certain actions, such as user interface gestures, which are mapped to actions in the real world and are used to work with the virtual world or model created, thus it engages the back integrative cortex. Most of the activity carried out by learners in order to explore, solve problems and request information is managed by this module. As the ILE should try to ensure that the learner has sufficiently automated key core knowledge or tasks in order to avoid unnecessary frustration those, activities must be familiar to the learner. This completely concurs with Vygostsky's Zone of Proximal Development and Piaget's concept of scaffolding.

\section{Conclusions}

Although the development of ILE does indeed seem to be a mayor undertaking, we show that a minimalist approach, one that may not need the use of complex visualization techniques and interaction systems may still produce extraordinary results. The specific weights that most be allocated to each phase of the learning cycle, the relation between these obviously mainly individual-learning environments 
and collaborative learning strategies, and the importance of the different kinds of cognitive load must further be studied and addressed.

\section{References}

[1] V. Alexandrov, N. Alexandrov and R. Ramirez-Velarde. "Novel Pedagogical Paradigms facilitating mixed mode and collaborative learning using open source software". International Conference on Interactive Computer Aided Learning ICL 2005, Villach, Austria, September, 2005.

[2] P. Dettmer. "New Blooms in Established Fields: Four Domains of Learning and Doing”. Roeper Review 28 p. 70-78, 2002. Academic Search Premier. EBSCO. 10 Mar. 2006.

[3] D. Berger-Ehrlich. "Establishing Connections: Interactivity Factors for a Distance Learning Education”. Educational Technology \& Society 5, 2002.

[4] J. J. G. Van Merriënboer, P. A. Kirschner \& L. Kester. "Taking the load of a learners' mind: Instructional design for complex learning”. Educational Psychologist, 38(1), p. 513, 2002.

[5] J. Zull. "The Art of Changing the Brain”. Stylus Publishing, Sterling, USA, 2002.

[6] Kolb and D. A. Kolb. "Experiential Learning Theory Bibliography 1971-2001". McBer and Co, Boston, USA, 2001.

[7] D. Wright. "The Moral Animal”. Vintage, Reprint edition, U.S.A., 1995.

[8] R. Ramírez-Velarde. "New Science for e-Learning Educational Models". Escuela de Tecnologia de Informacion de la Universidad de Xalapa, Xalapa, Mexico, October, 2005.

[9] P. A. Kirschner. "Cognitive load theory: implications of cognitive load theory on the design of learning”. Learning and Instruction, Volume 12, Issue 1, p. 1-10, 2002.

[10] J. M. van Bruggen, P. A. Kischner and W. Jochems. "External representation of argumentation in CSCL and the management of cognitive load". Learning and Instruction, 12, p. 121-138, 2002.

[11] B. Shneiderman. "Designing the User Interfase". Addison-Wesley Company Publishing Inc. 1987.

[12] G. G. Robertson, J. D. Mackinlay, S. K. Card. "Cone Trees: animated 3D visualizations of hierarchical information”. Proceedings of the SIGCHI conference on Human factors in computing systems, p.189-194, April 27-May 02, 1991, New Orleans, Louisiana, U.S.A.

[13] J. D. Mackinlay, G. G. Robertson and S. K. Card, "The perspective wall: detail and context smoothly integrated". Proceedings of the SIGCHI conference on Human factors in computing systems: Reaching through technology, p.173-176, April 27-May 02, 1991, New Orleans, Louisiana, United States

[14] S. K. Card, G. G. Robertson, and J. D. Mackinlay. "The information visualizer, an information workspace”. In Proceedings of ACM CHI'91 Conference on Human Factors in Computing Systems, Information Visualization, pages 181-188, 1991.

[15] D. A. Bowman, E. Kruijff, J. J. LaViola and I. Poupyrev. "3D User Interfaces: Theory and Practice”. Addison-Wesley Professional, July 26, 2004.

[16] S. Sorden. "A Cognitive Approach to Instructional Design for Multimedia Learning”. Informing Science Journal Volume 8, p. 263-279, 2005

[17] J. R. Brown and S. Cunningham. "Visualization in Higher Education". Academic Computing, 4 (6), p. 24-25, 41-45, 1990.

[18] S. Cunningham and W. Zimmerman (Editors). "Visualization in Teaching and Learning Mathematics". Mathematical Association of America Notes, N. 19, 1991.

[19] R. Ellson. "Visualization at Work". Academic Computing, p. 26, March 1990.

[20] R. C. Clark and R. E. Mayer. "e-Learning and the Science of Instruction”. Pfeiffer, N.Y., U.S.A., 2003. 